\title{
Impulsivity and cigarette craving: differences across subtypes
}

\author{
Neal Doran • Jessica Cook • Dennis McChargue • \\ Bonnie Spring
}

Received: 29 May 2009 / Accepted: 27 August 2009/Published online: 16 September 2009

(C) The Author(s) 2009. This article is published with open access at Springerlink.com

\begin{abstract}
Rationale Cigarette smoking has been linked to a number of personality characteristics, including impulsivity. Smokers tend to endorse high levels of impulsivity, and more impulsive smokers have greater difficulty quitting, but little is known about potential explanatory mechanisms. Although indirect evidence suggests craving as a candidate mechanism, direct evidence has been mixed.

Materials and methods This study assessed whether specific aspects of impulsivity (sensation seeking, lack of premeditation, lack of perseverance, and urgency) were associated with cue-induced craving. Regular smokers $(n=$ $60 ; 50 \%$ female) were exposed to a smoking cue and a neutral cue in a repeated measure counter-balanced design. Results Mixed effects regression models indicated that smokers who were high in sensation seeking reported greater increases in appetitive craving after smoking cue
\end{abstract}

N. Doran

Department of Psychiatry, University of California, San Diego,

San Diego, California, USA

J. Cook

Department of Medicine, University of Wisconsin,

Madison, Wisconsin, USA

D. McChargue

Department of Psychology, University of Nebraska-Lincoln, Lincoln, Nebraska, USA

B. Spring

Department of Preventive Medicine, Northwestern University, Chicago, Illinois, USA

\section{N. Doran $(\bowtie)$}

Department of Psychiatry, University of California, San Diego,

VMRF room 317, 3350 La Jolla Village Drive,

La Jolla, California 92161, USA

e-mail: nmdoran@ucsd.edu exposure, whereas, smokers who were high in urgency and lack of perseverance reported greater increases in negative affect craving.

Conclusions Findings suggest a complex relationship between impulsivity and cue-induced craving that may be masked by single construct conceptualizations of impulsivity.

Keywords Nicotine $\cdot$ Addiction · Behavior · Disinhibition

\section{Introduction}

A number of maladaptive personality characteristics have been found to be more prevalent among cigarette smokers relative to the general population (Gilbert and Gilbert 1995). That is, those who later begin smoking may be particularly likely to possess preexisting psychological and biological characteristics that increase their risk of initiation and inhibit their ability to quit. Much of the research in this area has focused on traits primarily associated with negative affect, such as depression proneness (Hitsman et al. 2003), neuroticism (Kirk et al. 2001; Terracciano and Costa 2004), hostility (Weiss et al. 2005), trait anxiety, and anxiety sensitivity (Comeau et al. 2001). However, recent research indicates that traits associated with appetitive, rewardseeking behavior, such as impulsivity, also influence tobacco use (Bickel et al. 1999; Doran et al. 2004; Mitchell 2004).

While impulsivity has long been recognized as an important contributor to smoking and other maladaptive behaviors (Williams 1973), research has been hampered by the lack of a consistent definition of impulsivity. Definitions have included lack of forethought, restlessness, difficulty delaying gratification, preference for immediate rewards, and a tendency toward risky behavior, among others (Evenden 1999; Mitchell 2004). Ambiguity surrounding the definition 
of impulsivity has been further complicated by use of alternate labels for equivalent constructs, such as disinhibition or constraint (Whiteside and Lynam 2001). More recent research indicates that impulsivity is a multidimensional construct. A factor analysis of 20 commonly used self-report measures of impulsivity yielded four factors: lack of premeditation regarding the consequences of behavior, lack of perseverance when tasks are boring or aversive, sensation seeking, and urgency, or the tendency to behave impulsively while experiencing negative affect (Whiteside and Lynam 2001). It has been suggested that the different facets of impulsivity are related but distinct constructs with different neurobiological and environmental underpinnings that act in concert to influence risky, maladaptive behavior (Lejuez et al. 2005). To the extent that this model is accurate, it is plausible that the specific component or components that determine an impulsive act are dependent upon the environmental context.

Smokers have consistently been found to be more impulsive than nonsmokers (Kassel et al. 1994; Mitchell 1999). Additionally, several recent studies suggest that more impulsive smokers have greater difficulty quitting (Covey et al. 2008; Doran et al. 2004; Kahler et al. in press). While relatively little is known about the nature of the relationship between impulsivity and smoking, recent research has begun to examine possible explanatory mechanisms. For example, more impulsive smokers have been shown to both expect (Doran et al. 2007a; VanderVeen et al. 2008a) and subjectively receive (Doran et al. 2006; Perkins et al. 2008) greater reinforcement from smoking.

These findings raise the possibility of a relationship between impulsivity and cigarette craving. To the extent that impulsive smokers both anticipate and perceive that they get greater reinforcement from smoking, they might be expected to experience stronger craving in response to external (e.g., smoking advertisements and other smokers) and internal (e.g., withdrawal and negative affect) cues. In other words, because they have a stronger belief that cigarettes will provide pleasurable experiences and alleviate aversive ones, they may have a stronger urge to smoke after exposure to a smoking cue. This hypothesis is consistent with previous reports of a positive association between smoking reinforcement expectancies and cue-induced craving (Brandon et al. 1996; Palfai 2002). It is also consistent with studies demonstrating a correlation between impulsivity and craving among individuals dependent on other substances, including alcohol (Zilberman et al. 2003) and opiates (Powell et al. 1992).

Cigarette craving is often examined in cue reactivity studies, which are based on classical conditioning processes. It is thought that repeated pairing with smoking results in certain environmental cues serving as conditioned stimuli, eliciting the urge to smoke (Carter and Tiffany
1999; Tiffany 1995). Such studies have exposed smokers to different cue modalities, including cigarettes or other smoking paraphernalia, audio- and videotapes with smoking-related content, and standardized and idiographic scripts related to smoking and have consistently produced large increases in self-reported cigarette craving $(d=1.18$ (Carter and Tiffany 1999)). Although the idea has received little attention in the cue reactivity literature, individual differences in smoking cue reactivity may exist. Two recent reports suggest that craving reactivity to smoking cues is stable over three (Miranda et al. 2008) or four (LaRowe et al. 2007) cue exposure sessions, approximately 1 week apart. Additionally, a study of 129 recent quitters compared their responses to in vivo cues and scripted exposure to a high-risk situation, a situation associated with a recent relapse, and a negative affect situation. Correlations between self-reported craving across modalities ranged from 0.32 to 0.70 (Niaura et al. 1998), indicating that individual smokers may respond relatively similarly across cue modalities. It is possible that individual differences in cue reactivity are a consequence of other individual differences, such as impulsivity, that may be associated with cigarette craving and other smoking variables.

Despite the apparent basis for positing a link between impulsivity and cigarette craving, findings have been mixed. In a survey study of 40 light-to-moderate smoking college students (Billieux et al. 2007), urgency was positively correlated with cravings associated with anticipating relief from negative affect but not with appetitive craving (i.e., craving and anticipating pleasure from smoking); lack of premeditation, lack of perseverance, and sensation seeking were not related to either craving subtype. A second study found a positive relationship between a composite measure of impulsivity (Patton et al. 1995)and a single item measure of craving that was averaged over $48 \mathrm{~h}$ of nicotine deprivation (VanderVeen et al. 2008b). However, a similar study found that the BIS-11 was not associated with average composite craving scores over the first 48 hours of a quit attempt (Doran et al. 2004). Finally, one study utilizing a cue reactivity procedure showed that the BIS-11 predicted greater increases in composite cigarette craving following exposure to an environmental smoking cue (Doran et al. 2007b), whereas another cue reactivity study using the same measures found no effect (Doran et al. 2008a).

These mixed findings may be a function of imprecise measurement of both impulsivity and craving. Of the five studies cited above, four used composite measures of impulsivity rather than assessing the components of the construct; the one study that did assess impulsivity components (Billieux et al. 2007) was a survey study and thus, may not reflect the experience of craving in general (i.e., in response to smoking cues). Along the same lines, several of 
the studies used mean craving ratings at pre-specified time points over a 48-h period as their primary craving outcome. However, craving is typically conceptualized as a state response to either internal (e.g., affective) or external (e.g., situations in which one typically smokes) cues (Tiffany et al. 2008). As such, it is not surprising that craving ratings that are averaged and are not in response to cues would produce inconsistent results.

The present study was designed to address these measurement issues by testing the relationship between impulsivity and cigarette craving in the context of a more precise assessment of impulsivity. To more accurately reflect the experience of smokers outside the laboratory, we used a cue reactivity paradigm to elicit craving. In addition to examining components of impulsivity (sensation seeking, urgency, lack of premeditation, and lack of perseverance) separately, we measured two craving subtypes: appetitive craving, reflecting craving that anticipates pleasure from smoking, and negative affect craving, which reflects craving that anticipates relief from aversive states via smoking (Cox et al. 2001). We hypothesized that the impulsivity component associated with reward-seeking behavior (sensation seeking) would predict increased appetitive craving following exposure to a smoking cue. Similarly, we expected that the components associated with impulsive actions in the context of negative affect (urgency and lack of perseverance) would predict increased negative affect craving in response to smoking cue exposure.

\section{Method}

Sample

Participants $(n=60 ; 50 \%$ male $)$ were regular smokers recruited from the community aged 18 to $65(M=30.8$; standard deviation $(\mathrm{SD})=10.8)$. They were required to have smoked at least 15 cigarettes per day $(M=19.1, \mathrm{SD}=5.2)$ for at least one year $(M=18.1, \mathrm{SD}=9.9)$, and to not meet criteria for any axis I disorder other than nicotine dependence. Participants were moderately nicotine dependent, with an average of $5.4(\mathrm{SD}=1.9)$ on the Fagerstrom Test for Nicotine Dependence (FTND; (Heatherton et al. 1991)). The sample was diverse, with $51 \%$ reporting Caucasian ethnicity and 33\% African-American. Participants were recruited using flyers distributed around the campus of a large, public, Midwestern university and the surrounding community, and were paid $\$ 70$ to participate in one screening and two experimental sessions. The study was approved by the university's Institutional Review Board, and all participants provided written informed consent.
Measures

Impulsivity Impulsivity was measured at the baseline screening session using the UPPS Impulsiveness Questionnaire (Whiteside and Lynam 2001). The UPPS is a 45 -item self-report measure composed of four subscales: urgency (e.g., "When I feel bad, I will often do things I later regret in order to make myself feel better for now."), sensation seeking (e.g., "I quite enjoy taking risks."), lack of perseverance (e.g., "I tend to give up easily."), and lack of premeditation (e.g., "My thinking is usually careful and purposeful."). Each subscale is scored so that higher values reflect greater impulsivity. The UPPS has good internal consistency, with Cronbach's alpha coefficients in this sample ranging from 0.78 to 0.90 for the four subscales. Correlations between UPPS subscales are typically modest (e.g., $r=0.22$; (Whiteside and Lynam 2001)), suggesting that the subscales index distinct components of impulsivity; the average correlation between subscales in the present study was $0=0.26$.

Cigarette craving Craving was measured with the brief version of the Questionnaire of Smoking Urges (QSU-Brief; (Cox et al. 2001)), a 10-item self-report measure. The QSUBrief contains two subscales: a five-item appetitive craving subscale, reflecting craving that anticipates pleasure, and a four-item negative affect craving subscale, reflecting cravings that anticipate relief from aversive states. Respondents rate each item on a scale from 0 to 100 , and subscale scores are calculated by averaging item ratings. The QSU-Brief has good construct validity and reliability (Cox et al. 2001). Internal consistency in the present study ranged from 0.84 to 0.93 across measurement points. The QSU-Brief was administered twice during each experimental session: at baseline and immediately following cue exposure.

Smoking characteristics The FTND (Heatherton et al. 1991) was used at the screening session to assess nicotine dependence. This 6-item scale sums characteristics consistent with nicotine dependence. Scores can range from 0 to 10 , with higher scores reflecting increased dependence. In addition, we used assessments of expired carbon monoxide (CO) via ecolyzer (EC-50, Vitalograph Corp., Lenexa, Kansas, USA) at screening and each experimental session to confirm participants' report of their smoking status. We considered $\mathrm{CO}$ values $\geq 8 \mathrm{ppm}$ to be consistent with regular smoking. Two participants had values $<8$ ppm upon arriving for an experimental session. In each case the participant had misunderstood the instruction to smoke as usual and had refrained from smoking since waking on the day of the session. Both were rescheduled and instructed to smoke as usual before the rescheduled session, and had $\mathrm{CO}$ values $>8 \mathrm{ppm}$ at baseline of the new session. Finally, upon 
arriving for each experimental session, participants were asked when they had last smoked. Time since last cigarette ranged from 5 to $540 \mathrm{~min}(M=28.3, \mathrm{SD}=67.1$, median= 20). One participant had last smoked on the night prior to the session, while all others had last smoked within $90 \mathrm{~min}$ of the session.

Axis I disorders To determine whether candidates met criteria for current Axis I disorders other than nicotine dependence, trained interviewers administered the Structured Clinical Interview for DSM-III-R-Nonpatient Edition (SCID-NP; (Spitzer et al. 1992), which has been shown to compare favorably with other methods of diagnosis (Williams et al. 1992). Interviewers were supervised by a licensed clinical psychologist to ensure accuracy and reliability of diagnoses.

\section{Procedure}

Screening Candidates were first screened by telephone, at which point they provided demographic and smoking information for an initial assessment of eligibility. Those who were interested and eligible were then scheduled for an in-person screening interview. After the participants provided written informed consent, the SCID-NP was administered, and participants completed the UPPS, as well as questionnaires on demographics and smoking history. They were then scheduled for two experimental sessions.

Experimental sessions To increase generalizability, participants were instructed to smoke as usual prior to each session. Sessions were administered in counter-balanced order, and each 2-h session began between 8 a.m. and 10 a. $\mathrm{m}$. to minimize variance due to diurnal variance in craving (Tiffany et al. 2008). Each session began with the assessments of expired $\mathrm{CO}$, time since last cigarette, and craving. These assessments were followed by a 20 -min rest period to minimize the possibility that cue reactivity would be limited among participants who had smoked shortly before the session. Participants were then exposed to either a neutral cue or a smoking cue, after which craving was reassessed.

Cue exposures were conducted in the same room as the rest of the experimental sessions. The room was a laboratory office approximately $2.7 \mathrm{~m} \times 3.7 \mathrm{~m}$, and contained a desk for the experimenter and a table at which participants sat. Smoking paraphernalia not needed for the session were stored out of sight. The experimenter sat in the room while participants completed initial questionnaires but not during the rest period. The experimenter then returned and gave verbal instructions for the cue reactivity procedure, then left participants alone in the room for the duration of the 5-min exposure. An air filtration system (Hastings Air Energy Control, New Berlin, Wisconsin,
USA) was used between sessions to reduce the smell of smoke in the room.

Cue exposure The cue reactivity procedure was adapted from the one described by Sayette and Hufford (Sayette and Hufford 1994) and has been used in other cue reactivity research (Doran et al. 2008b). In the smoking cue condition, participants were given a new pack of their usual brand of cigarettes, and sat at the table with a lighter and ashtray in front of them. They were instructed to light a cigarette without touching it to their mouths, and to hold it in their hands for $5 \mathrm{~min}$ as if they were smoking, but without actually doing so. The neutral cue exposure was identical, except that the lighter and ashtray were not present, and participants were instructed to hold a roll of tape rather than a cigarette.

\section{Analytic plan}

Craving was assessed at four points (pre- and post-exposure in both cue conditions) using mixed effects regression modeling. By including baseline values in the time-varying dependent variable, this approach accounted for any baseline differences in craving. We utilized models with random intercepts, linear trends, and autoregressive errors; as recommended (Verbeke and Molenberghs 2000), this variance-covariance structure was chosen after comparing it with several other possible structures. We tested the effects of each of the four impulsivity components individually on both craving subtypes, yielding a total of eight regression models evaluated. Each model included age and gender, which are typically correlated with impulsivity (Eysenck et al. 1985; Waldeck and Miller 1997), as well as nicotine dependence, as covariates. Other potential covariates (e.g., time since last cigarette and session order) were not included because they were not significantly correlated with impulsivity or to craving. Each model also included time and cue condition, as well as the three-way interaction between those variables and the impulsivity variable in the model. As recommended in the statistical literature (Peixoto 1987; West et al. 1996), all lower order two-way interactions between time, condition, and impulsivity were also included. Significant impulsivity $\times$ time $\times$ condition terms indicated that the post-exposure change in craving varied as a function of both condition and the impulsivity variable.

The mixed effects approach is preferable to other methods (e.g., repeated measures ANOVA) because it allows for the inclusion of categorical and continuous predictors and of time-varying and time-invariant predictor and outcome variables in a single model. Additionally, the autoregressive variance-covariance structure makes assumptions about the correlation between repeated measurements of a single variable (i.e., craving in the present study) that are more 
realistic. While ANOVA assumes that all assessments of a repeated measure variable are similarly correlated, mixed effects regression with autoregressive errors assumes that the correlations vary as a function of their temporal proximity (Gibbons et al. 1993; Hedeker et al. 1996). Finally, univariate methods estimate change over time averaged across participants, whereas mixed effects regression estimates change for each participant. Given that the rate of change is likely to vary across individuals, the latter are likely a more accurate reflection of the data (Hedeker 2002).

\section{Results}

Preliminary analyses

To test the validity of the cue exposure, we first used mixed effects regression to assess pre- to post-exposure changes in appetitive craving and in negative affect craving (Fig. 1).

Findings indicated significant time (pre versus post) $\times$ condition (neutral versus smoking cue) interactions for both appetitive $(z=3.32, p=0.001)$ and negative affect $(z=2.35$, $p=0.019)$ craving. Simple effects tests indicated that participants reported a slight but nonsignificant increase in both craving subtypes after exposure to the neutral cue, and a significant increase in both after exposure to the smoking cue. Effect sizes for post-cue change in the smoking cue condition (appetitive craving, $d=1.26$; negative affect craving, $d=1.17$ ) were consistent with previous studies of smoking cue reactivity (Carter and Tiffany 1999).

\section{Primary analyses}

Appetitive craving Mixed effects regression models were used to separately test the effects of each impulsivity variable (urgency, sensation seeking, lack of premeditation, and lack of perseverance) on change in appetitive craving following cue exposure. In each model, the outcome variable consisted of the four assessments of appetitive

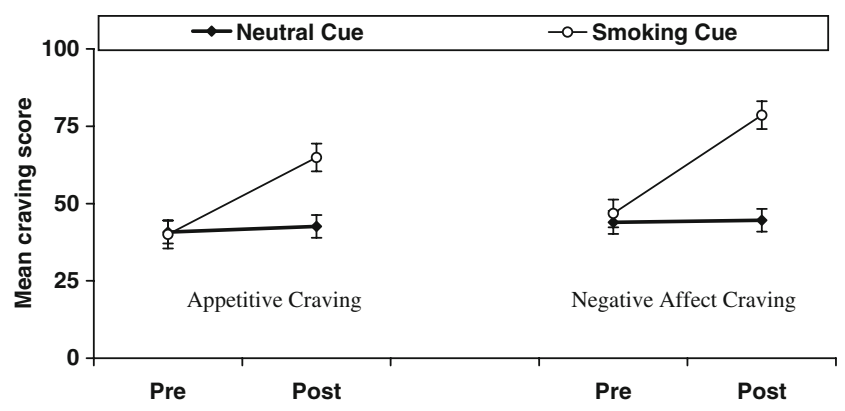

Fig. 1 Mean appetitive and negative affect craving by time (preversus post-cue exposure) and condition (neutral cue versus smoking cue) craving (i.e., pre- and post-exposure in both cue conditions) to account for baseline levels of craving. Male gender and higher levels of nicotine dependence, but not age, were associated with higher levels of craving at each assessment. Urgency, lack of premeditation, and lack of perseverance were all unrelated to post-exposure changes in appetitive craving (see Table 1).

However, in the sensation seeking model, we found a significant sensation seeking $\times$ time $\times$ condition interaction $[t(118)=2.28, p=0.02$; Table 1]. Simple effects tests via linear regression revealed that after controlling for baseline appetitive craving, higher levels of sensation seeking were associated with greater post-exposure increases in appetitive craving in the smoking cue condition $(\beta=0.33, p=0.02)$ but not the neutral cue condition. Because sensation seeking and appetitive craving were each measured continuously, to depict the relationship graphically, we plotted correlations between the two by time and condition. As Fig. 2 indicates, there was a modest but significant association between sensation seeking and appetitive craving at baseline in both conditions. The strength of the correlation increased substantially after exposure to a smoking cue but not a neutral cue, indicating that participants who were higher in sensation seeking had a stronger appetitive craving response to the smoking cue than others.

Negative affect craving Similarly, four mixed effects regression models were used to test the effect of each impulsivity variable on changes in negative affect craving following cue exposure. The dependent variable in each model consisted of the four assessments of negative affect craving. Higher levels of nicotine dependence, but not age or gender, predicted stronger negative affect craving at each measurement. The models for sensation seeking and lack of

Table 1 Mixed effects regression model of the effect of sensation seeking on appetitive craving by time and cue condition

\begin{tabular}{lrrc}
\hline Effect & Estimate & Std err & T value \\
\hline Intercept & 12.09 & 4.04 & $3.00^{* *}$ \\
Age & 10.27 & 11.41 & 0.90 \\
Gender & -2.56 & 1.24 & $-2.06^{*}$ \\
FTND & 1.62 & 0.74 & $2.19^{*}$ \\
Time & -0.15 & 1.02 & -0.15 \\
Condition & -2.77 & 3.06 & -0.91 \\
Time x condition & 0.29 & 0.27 & 1.07 \\
Sensation seeking & 0.33 & 0.13 & $2.47^{*}$ \\
Sensation seeking x time & 0.06 & 0.06 & 1.04 \\
Sensation seeking x condition & 0.18 & 0.14 & 1.28 \\
Sensation seeking x time x condition & 0.19 & 0.09 & $2.28^{*}$ \\
\hline
\end{tabular}

FTND Fagerstrom test for nicotine dependence ${ }^{*} p<0.05 ;{ }^{* *} p<0.01$ 


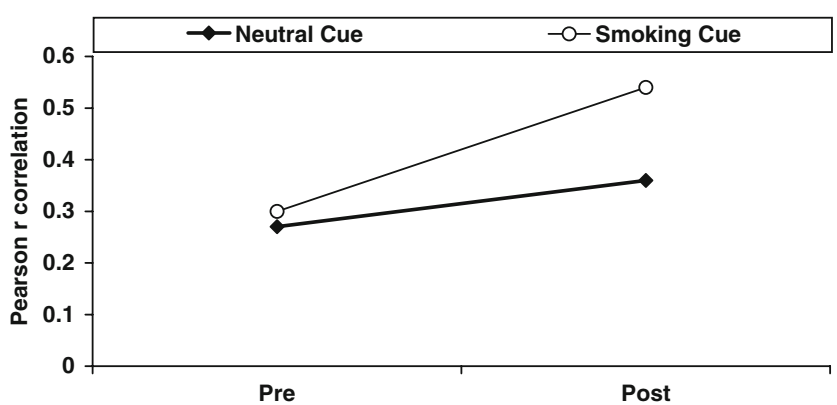

Fig. 2 Correlations between sensation seeking and appetitive craving by time (pre- versus post-cue exposure) and condition (neutral cue versus smoking cue)

premeditation indicated that these variables were not associated with differential changes in negative affect craving after cue exposure (see Table 2).

The urgency model yielded a significant urgency $\times$ time $\times$ condition effect $[t(118)=3.77, p<0.01$; Table 2]. Simple effects tests indicated that controlling for baseline negative affect craving, higher urgency was associated with greater change in negative affect craving in the smoking cue condition $(\beta=.45, p=0.004)$ but not the neutral cue condition. The model for lack of perseverance similarly indicated a significant lack of perseverance $\times$ time $\times$ condition effect $[t(118)=2.52, p=0.010$; Table 3]. Simple effects tests controlling for negative affect craving at baseline showed that, like urgency, participants with greater difficulty with perseverance reported greater changes in negative affect craving after exposure to the smoking cue $(\beta=0.40, p=$ 0.001 ) but not the neutral cue. To describe these effects graphically, we again plotted correlations between impulsivity variables and craving by time and cue condition. As shown in Fig. 3, negative affect craving had a significant but small correlation with both urgency and lack of perseverance

Table 2 Mixed effects regression model of the effect of urgency on negative affect craving by time and cue condition

\begin{tabular}{lccc}
\hline Effect & Estimate & Std err & T value \\
\hline Intercept & -2.31 & 3.32 & -0.70 \\
Age & -3.56 & 2.88 & 1.24 \\
Gender & 0.47 & 0.52 & 0.90 \\
FTND & 1.49 & 0.72 & $2.07^{*}$ \\
Time & 4.75 & 1.53 & $3.10^{* *}$ \\
Condition & 2.31 & 2.08 & 1.11 \\
Time x condition & 0.17 & 0.23 & 0.74 \\
Urgency & 0.20 & 0.09 & $2.22^{*}$ \\
Urgency x time & 0.21 & 0.10 & $2.15^{*}$ \\
Urgency x condition & 0.03 & 0.07 & 0.46 \\
Urgency x time x condition & 0.12 & 0.03 & $3.77^{* *}$ \\
\hline
\end{tabular}

FTND Fagerstrom test for nicotine dependence

${ }^{*} p<0.05 ; * * p<0.01$
Table 3 Mixed effects regression model of the effect of lack of perseverance on negative affect craving by time and cue condition

\begin{tabular}{lrrc}
\hline Effect & Estimate & Std err & T value \\
\hline Intercept & 7.62 & 4.55 & 1.67 \\
Age & -1.12 & 1.72 & -0.65 \\
Gender & -0.10 & 0.13 & -0.73 \\
FTND & 1.34 & 0.62 & $2.16^{*}$ \\
Time & 0.98 & 1.69 & 0.58 \\
Condition & -0.45 & 2.22 & -0.20 \\
Time x condition & 0.17 & 0.14 & 1.21 \\
Lack of perseverance & 0.32 & 0.13 & $2.36^{*}$ \\
Lack of perseverance x time & 0.07 & 0.14 & 0.50 \\
Lack of perseverance $x$ condition & 0.08 & 0.08 & 1.07 \\
Lack of perseverance $x$ time x condition & 0.08 & 0.03 & $2.52^{*}$ \\
\hline
\end{tabular}

FTND Fagerstrom test for nicotine dependence

${ }^{*} p<0.05 ;{ }^{* *} p<0.01$

at baseline in both conditions. The strength of these relationships increased after exposure to the smoking cue but not the neutral cue, indicating that participants higher in the urgency and lack of perseverance components of impulsivity had a greater negative affect craving response to the smoking cue than others.

\section{Discussion}

The present study tested the hypothesis that the effect of impulsivity on cigarette craving following exposure to a smoking cue would vary as a function of the impulsivity and craving subtypes being measured. We found that, as hypothesized, sensation seeking was associated with a greater appetitive craving response to a smoking cue, whereas urgency and lack of perseverance were associated with a greater negative affect craving response. The final impulsivity subtype, lack of premeditation, did not predict appetitive or negative affect craving response to cue exposure.

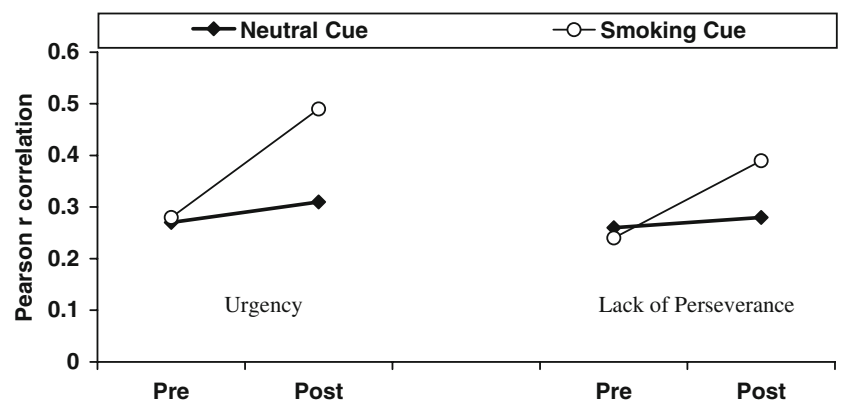

Fig. 3 Correlations of urgency and lack of perseverance with negative affect craving by time (pre- versus post-cue exposure) and condition (neutral cue versus smoking cue) 
The literature on the relationship between impulsivity and cigarette craving has been mixed, with only some studies finding a significant relationship. Our results suggest that the inconsistency of previous results may be due to inconsistencies of measurement, in that previous studies typically either conceptualized both impulsivity and craving as unidimensional constructs, or did not assess craving in response to a smoking cue, or both. Impulsivity is now generally recognized as being composed of several related but distinct constructs (Whiteside and Lynam 2001). Additionally, cue-induced craving procedures in the laboratory may better reflect the experiences of smokers outside the laboratory in comparison with previous measurement approaches. Consequently, it is plausible that design differences in the present study allowed us to more precisely assess the relationship between impulsivity and craving.

Our findings are consistent with conceptual explanations of impulsivity subtypes. For example, individuals high in sensation seeking may be accustomed to engaging in approach behavior when they encounter a cue that a reward is available; that is, sensation seeking smokers may be especially likely to smoke in response to a cigarette cue. When they are prevented from smoking after cue exposure, as in the present study, it is plausible that those high in sensation seeking would experience a greater urge to smoke. Because they tend to be motivated by the pursuit of positive reinforcement (Whiteside and Lynam 2001), it follows that such craving would reflect anticipation of pleasure from smoking. Smokers with high levels of urgency and lack of perseverance may similarly be more accustomed than others to smoking in response to cues, but with different motivations. Specifically, they may tend to experience heightened negative affect after being exposed to smoking cues (Doran et al. 2008b). To the extent that they perceive smoking as being more negatively reinforcing than other smokers do, these individuals may tend to experience cue-induced urges to smoke that reflect a desire to ameliorate negative affect.

As hypothesized, the lack of premeditation aspect of impulsivity was not associated with cue-induced craving. This is consistent with previous research, which suggests that lack of premeditation is associated with cigarette consumption (Miller et al. 2003) but not with craving (Billieux et al. 2007). Lack of premeditation is conceptualized as failure to consider the potential negative consequences of a behavior prior to engaging in it (Whiteside and Lynam 2001). Individuals who lack premeditation may therefore be more likely to smoke or to smoke more frequently than others because they are less likely to be deterred by the negative effects of smoking. For the same reason, smokers who lack premeditation may be particularly likely to respond to cigarette craving by smoking. However, the tendency not to consider the consequences of one's behavior may only influence constructs that reflect explicit behaviors, unlike cigarette craving.

Some aspects of this study may limit its generalizability. First, craving was induced via a standardized cue presented in a laboratory environment. It is possible that participants responded differently to the cue exposure procedure compared with their responses to environmental smoking cues outside the laboratory. Second, individuals currently meeting criteria for axis I disorders other than nicotine dependence were excluded. Because psychological disorders and symptoms are more common among both smokers (Hughes 1999) and more impulsive individuals (Moeller et al. 2001), the sample used here may not be representative of impulsive smokers in general. Finally, our measurement approach may have failed to capture some constructs that are relevant to impulsivity. For example, recent research suggests that two types of urgency exist: negative urgency, as described above, and positive urgency, or the tendency toward impulsive behavior under conditions of extreme positive affect. Evidence suggests that both aspects of urgency contribute to risky behaviors such as smoking (Cyders and Smith 2008).

In sum, our findings indicate that the relationship between impulsivity and cue-induced cigarette craving is complex and varies as a function of subtypes of both variables. Notably, smokers who were prone to impulsive behavior in pursuit of positive reinforcement (i.e., sensation seeking) tended to respond to the smoking cue with appetitive cravings, whereas those prone to impulsiveness associated with avoidance of negative affect responded with negative affect cravings. These data provide further evidence that impulsivity is a multi-faceted construct and that failure to account for this may obscure the relationship between impulsivity and other variables. While these findings add to our knowledge of the relationship between impulsivity and cigarette smoking, further research is needed to fully explain the relationship. For example, if urgency and sensation seeking produce different responses to a standardized smoking cue, it may be useful to examine whether they are associated with varied response to different cues (e.g., negative affective versus positive affective cues). Additionally, while recent research has indicated that impulsivity is composed of multiple related but distinct constructs, to our knowledge no research has examined whether "impulsivity profiles" can be derived - that is, whether some aspects of impulsivity hang together more than others, and if so how they may influence smoking and other risky behaviors. Finally, it is not currently clear whether impulsivity is a risk factor for smoking initiation and dependence or vice versa or both (Mitchell 2004). The answer to this question may have important implications for the understanding of the role of impulsivity in smoking development, as well as prevention and cessation efforts. 
Acknowledgments This study was supported by American Heart Association Award 0410025Z and by a New Investigator Award from the California Tobacco-Related Disease Research Program 17KT-0027.

Open Access This article is distributed under the terms of the Creative Commons Attribution Noncommercial License which permits any noncommercial use, distribution, and reproduction in any medium, provided the original author(s) and source are credited.

\section{References}

Bickel WK, Odum AL, Madden GJ (1999) Impulsivity and cigarette smoking: delay discounting in current, never, and ex-smokers. Psychopharmacology (Berl) 146:447-454

Billieux J, Van der Linden M, Ceschi G (2007) Which dimensions of impulsivity are related to cigarette craving? Addict Behav 32:1189-1199

Brandon TH, Wetter DW, Baker TB (1996) Affect, expectancies, urges, and smoking: do they conform to models of drug motivation and relapse? Exp Clin Psychopharmacol 4:29-36

Carter BL, Tiffany ST (1999) Meta-analysis of cue-reactivity in addiction research. Addiction 94:327-340

Comeau N, Stewart SH, Loba P (2001) The relations of trait anxiety, anxiety sensitivity, and sensation seeking to adolescents' motivations for alcohol, cigarette, and marijuana use. Addict Behav 26:803-825

Covey LS, Manubay J, Jiang H, Nortick M, Palumbo D (2008) Smoking cessation and inattention or hyperactivity/impulsivity: a post hoc analysis. Nicotine Tob Res 10:1717-1725

Cox LS, Tiffany ST, Christen AG (2001) Evaluation of the brief questionnaire of smoking urges (QSU-brief) in laboratory and clinical settings. Nicotine Tob Res 3:7-16

Cyders MA, Smith GT (2008) Emotion-based dispositions to rash action: positive and negative urgency. Psychol Bull 134:807828

Doran N, Spring B, McChargue D, Pergadia M, Richmond M (2004) Impulsivity and smoking relapse. Nicotine Tob Res 6:641-647

Doran N, McChargue D, Spring B, VanderVeen J, Cook JW, Richmond M (2006) Effect of nicotine on negative affect among more impulsive smokers. Exp Clin Psychopharmacol 14:287-295

Doran N, McChargue D, Cohen L (2007a) Impulsivity and the reinforcing value of cigarette smoking. Addict Behav 32:90-98

Doran N, Spring B, McChargue D (2007b) Effect of impulsivity on craving and behavioral reactivity to smoking cues. Psychopharmacology (Berl) 194:279-288

Doran N, Cook JW, McChargue D, Myers MG, Spring B (2008a) Cue-elicited negative affect in impulsive smokers. Psychol Addict Behav 22:249-256

Doran N, McChargue D, Spring B (2008b) Effect of impulsivity on cardiovascular and subjective reactvity to smoking cues. Addict Behav 33:167-172

Evenden JL (1999) Varieties of impulsivity. Psychopharmacology (Berl) 146:348-361

Eysenck SBG, Pearson PR, Easting G, Allsopp JF (1985) Age norms for impulsiveness, venturesomeness, and empathy in adults. Personality \& Individual Differences 6:613-619

Gibbons RD, Hedeker D, Elkin I, Waternaux C, Kraemer HC, Greenhouse JB (1993) Some conceptual and statistical issues in analysis of longitudinal psychiatric data: application to the NIMH treatment of depression collaborative research program dataset. Arch Gen Psychiatry 50:739-750

Gilbert DG, Gilbert BO (1995) Personality, psychopathology, and nicotine response as mediators of the genetics of smoking. Behav Genet 25:133-147
Heatherton TF, Kozlowski LT, Frecker RC, Fagerstrom KO (1991) The Fagerstrom test for nicotine dependence: a revision of the Fagerstrom tolerance questionnaire. Br J Addict 86:1119-1127

Hedeker D (2002) An introduction to growth modeling. In: Kaplan D (ed) Handbook of quantitative methodology for the social sciences. Sage, Thousand Oaks, CA

Hedeker D, Flay BR, Petraitis J (1996) Estimating individual influences of behavioral intentions: An application of random effects modeling to the theory of reasoned action. J Consult Clin Psychol 64:109-120

Hitsman B, Borrelli B, McChargue D, Spring B, Niaura R (2003) History of depression and smoking cessation outcome: A metaanalysis. J Consult Clin Psychol 71:657-663

Hughes JR (1999) Comorbidity and smoking. Nicotine Tob Res 1: S149-S152

Kahler CW, Spillane NS, Metrik J, Leventhal AM, Monti PM (2009) Sensation seeking as a predictor of treatment compliance and smoking cessation treatment outcomes in heavy social drinkers. Pharmacology, Biochemistry and Behavior 93:285-290

Kassel JD, Shiffman S, Gnys M, Paty J, Zettler-Segal M (1994) Psychosocial and personality differences in chippers and regular smokers. Addict Behav 19:565-575

Kirk KM, Whitfield JB, Pang D, Heath AC, Martin NG (2001) Genetic covariation of neuroticism with monoamine oxidase activity and smoking. Am J Med Genet 105:700-706

LaRowe SD, Saladin ME, Carpenter MJ, Upadhyaya HP (2007) Reactivity to nicotine cues over repeated cue reactvity sessions. Addict Behav 32:2888-2899

Lejuez CW, Aklin W, Bornovalova M, Moolchan ET (2005) Differences in risk-taking propensity across inner-city adolescent everand never-smokers. Nicotine Tob Res 7:71-79

Miller J, Flory K, Lynam D, Leukefeld C (2003) A test of the fourfactor model of impulsivity-related traits. Personality \& Individual Differences 34:1403-1418

Miranda R Jr, Rohsenow DJ, Monti PM, Tidey J, Ray L (2008) Effect of repeated days of smoking cue exposure on urge to smoke and physiological reactvity. Addict Behav 33:347-353

Mitchell SH (1999) Measures of impulsivity in cigarette smokers and non-smokers. Psychopharmacology (Berl) 146:455-464

Mitchell SH (2004) Measuring impulsivity and modeling its association with cigarette smoking. Behav Cogn Neurosci Rev 3:261-275

Moeller FG, Barratt ES, Dougherty DM, Schmitz JM, Swann AC (2001) Psychiatric aspects of impulsivity. Am J Psychiatry 158:1783-1793

Niaura R, Shadel WG, Abrams DB, Monti PM, Rohsenow DJ, Sirota A (1998) Individual differences in cue reactivity among smokers trying to quit: effects of gender and cue type. Addict Behav 23:209-224

Palfai TP (2002) Positive outcome expectancies and smoking behavior: the role of expectancy accessibility. Cogn Ther Res 26:317-333

Patton JH, Stanford MS, Barratt ES (1995) Factor structure of the Barratt impulsiveness scale. J Clin Psychol 51:768-774

Peixoto JL (1987) Hierarchical variable selection in polynomial regression models. Am Stat 41:311-313

Perkins KA, Lerman C, Coddington SB, Jetton C, Karelitz JL, Scott JA et al (2008) Initial nicotine sensitivity in humans as a function of impulsivity. Psychopharmacology (Berl) 200:529-544

Powell J, Bradley B, Gray J (1992) Classical conditioning and cognitive determinants of subjective craving for opiates: an investigation of their relative contributions. Br J Addict 87:1133-1144

Sayette MA, Hufford MR (1994) Effects of cue exposure and deprivation on cognitive resources in smokers. J Abnorm Psychol 103:812-818

Spitzer RL, Williams JB, Gibbon M, First MB (1992) The structured clinical interview for DSM-III-R (SCID). History, rationale, and description. Arch Gen Psychiatry 49:624-629 
Terracciano A, Costa PT Jr (2004) Smoking and the five-factor model of personality. Addiction 99:472-481

Tiffany ST (1995) Potential functions of classical conditioning in drug addiction. In: Drummond DC, Tiffany ST, Glautier S, Remington B (eds) Addictive behaviour: cue exposure theory and practice. John Wiley \& Sons, Chichester, England

Tiffany ST, Warthen MW, Goedeker KG (2008) The functional significance of craving in nicotine dependence. In 55th Nebraska Symposium on Motivation. Springer-Verlag, New York

VanderVeen J, Cohen LM, Cukrowicz KC, Trotter DRM (2008a) The role of impulsivity on smoking maintenance. Nicotine Tob Res 10:1397-1404

VanderVeen JW, Cohen LM, Trotter DRM, Collins FL (2008b) Impulsivity and the role of smoking-related outcome expectancies among dependent college-aged cigarette smokers. Addict Behav 33:1006-1011

Verbeke G, Molenberghs G (2000) Linear mixed models for longitudinal data. Springer, New York

Waldeck TL, Miller LS (1997) Gender and impulsivity differences in licit substance use. J Subst Abuse 9:269-275
Weiss JW, Mouttapa M, Chou C-P, Nezami E, Johnson CA, Palmer $\mathrm{PH}$ et al (2005) Hostility, depressive symptoms, and smoking in early adolescence. J Adolesc 28:49-62

West SG, Aiken LS, Krull JL (1996) Experimental personality designs: analyzing categorical by continuous variable interactions. J Pers 64:1-48

Whiteside SP, Lynam DR (2001) The five factor model and impulsivity: using a structural model of personality to understand impulsivity. Personality \& Individual Differences 30:669-689

Williams AF (1973) Personality and other characteristics associated with cigarette smoking among young teenagers. J Health Soc Behav 14:374-380

Williams JBW, Gibbon M, First MB, Spitzer RL, Davies M, Borus J et al (1992) The structured clinical interview for DSM-III-R (SCID). Multisite test-retest reliability. Arch Gen Psychiatry 49:630-636

Zilberman ML, Tavares H, el-Guebaly N (2003) Relationship between craving and personality in treatment-seeking women with substance-related disorders. BMC Psychiatry 3:1 doi:10.1186/ 1471-244X-3-1. Accessed July 28, 2009 from http://www. pubmedcentral.nih.gov/picrender.fcgi?artid=140309\&blobtype=pdf 